intramural/epicardial RMCC. Programmed ventricular stimulation assessed VT inducibility post procedure as a marker of immediate efficacy and device interrogations were conducted at $1,3,6$, and 12 months to determine time to VT recurrence.

Results 15 consecutive pts (median age 68yrs, LVEF 30\%) were studied (6 month pre-procedural ICD therapies: median 19 ATP events $(\mathrm{IQR}=4-93)$ and 1 shock (IQR=0-3)). Scar $(<1.5 \mathrm{mV})$ occupied a median $29 \%$ of the total surface area (median 523 scar points). A median of 2 RMCCs were seen within each scar (length 60mm; far field peak amplitude $0.43 \mathrm{mV}$; local $0.19 \mathrm{mV}$; conduction $53 \mathrm{~cm} / \mathrm{s}$ ). The Figure illustrates two RMCCs enhanced with design lines within a large anterior scar containing distinct late potentials ascending up the scar. This is readily apparent without manual annotation or point tagging. Ablation was performed along all RMCCs (median 19 lesions $(\mathrm{IQR}=10-23)$ ) and any non-connected sites (median 6 lesions $(\mathrm{IQR}=2-12)$. The diastolic isthmus in VT was mapped in 3 pts and co-located within the RMCCs identified. VT was non-inducible in $85 \%$ of patients post ablation. There were no complications during the procedures. In this cohort, 71\% remain free of VT recurrence at 6 month median follow up. Interestingly, during redo ablation in a patient with recurrence, an RMCC identified during the index procedure had not been completely eliminated.

Conclusions RM identifies conducting channels within the post-infarct scar that might support re-entrant VT and can be used to guide substrate based ablation to reduce future ICD therapies.

\section{A PHYSIOLOGIST LED LOOP RECORDER IMPLANTATION SERVICE IN AN AMBULATORY SETTING}

${ }^{1}$ Shabanna Din*, 'Sandra Belchambers, 'Susan Hughes, ${ }^{2}$ Jay Wright. ${ }^{1} \mathrm{LHCH} ;{ }^{2} \mathrm{LHVH}$; *Presenting Author

\subsection{6/heartjnl-2016-309890.69}

Our tertiary cardiac centre has developed a new ambulatory service for injectable loop recorder insertion based in a treatment room. Previously loop recorders were implanted in a dedicated pacing theatre by a consultant led team. The patient was kept nil by mouth prior to the procedure and were dressed in a theatre gown. The wound from the device was around $4 \mathrm{~cm}$ and was closed with sutures. The typical time in

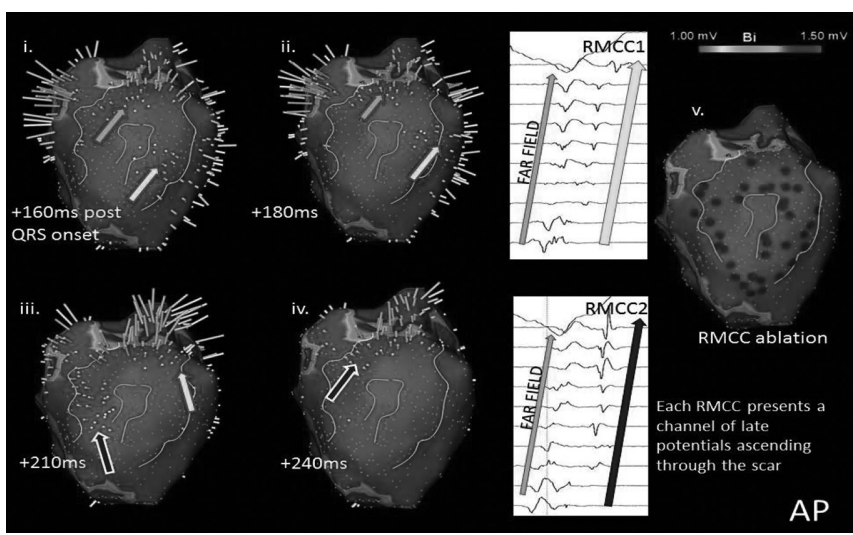

Abstract 68 Figure 1 The path of RMCC activation at 4 time points (i-iv) within scar, with associated electrograms and ablation lesions (v) theatre was around $30 \mathrm{~min}$. Patients were then observed for 2 $\mathrm{h}$ post procedure prior to discharge.

A new injectable reveal device became available with reduced surgical trauma and scarring. We subsequently developed a new service run by senior cardiac physiologists without the need for medical staff to be present. The physiologists were initially trained in obtaining consent, surgical skills and device implantation. They were then supervised for the first 20 implants before continuing independently. We looked retrospectively at the first 100 cases. The service was run from a treatment room on a day ward and was entirely ambulatory. Oral antibiotics and local anaesthetic were prescribed by a doctor prior to the implant. The average case time was less than $10 \mathrm{~min}$. Patients did not require fasting and were able to have the implant done in their own clothes. The wound is less than one $\mathrm{cm}$ and is closed with a surgical glue without the need for sutures. The patients were educated on use of the device and then discharged post procedure. The waiting time for the physiologist led service is significantly reduced with cases generally being done in under 2 weeks. Previously the waiting time for a consultant to implant a loop recorder was around 2 months. The adverse event rate is favourable with no implant site infections and 2 erosions. There have been no major adverse effects. The new service had freed up a considerable amount of theatre space and consultant time allowing a more efficient pacing service. The patient experience has been improved with a shorter time in hospital. The new device is more expensive than the previous loop recorder but the cost has been offset by the reduced staffing requirements and need for less equipment. We have demonstrated that implanting by non medical staff is safe, efficient and cost effective.

\section{A NOVEL AMBULATORY SYNCOPE ASSESSMENT UNIT IS SAFE AND COST-EFFECTIVE IN A LOW-RISK PATIENT COHORT}

${ }^{1}$ Hamish Mclachlan, ${ }^{1}$ Christopher Allen, ${ }^{1}$ Myura Nagendran, ${ }^{2}$ Gothandaraman Balaji* ${ }^{1} \mathrm{RBH}_{i}{ }^{2}$ Northwick Park Hospital; *Presenting Author

\subsection{6/heartjnl-2016-309890.70}

Introduction Syncope affects approximately 50\% of people in their lifetime. The diagnosis of the underlying cause for syncope is often delayed, inaccurate and cost-inefficient. ${ }^{1}$ The objective of this study was to evaluate the safety and costeffectiveness of a novel low-risk syncope day-case assessment unit recently introduced at a DGH.

Methods A retrospective analysis of 50 in-patients admitted with syncope was initially undertaken. Measured variables included length of in-patient admission, waiting time to investigation (24-hour holter and transthoracic echocardiogram), frequency of cardiology review, 30-day re-admission rate with syncope and 90-day mortality rate. The same variables were then assessed prospectively in 50 in-patients referred directly to the syncope clinic. The one-stop assessment unit took place in the local ambulatory care unit (Figure 1) and was led by acute medical physicians; with access to transthoracic echocardiography and continuous ECG monitoring which were all performed on the same day. Patients were referred for sameday specialist review as appropriate. All 100 patients were deemed low risk as defined by the San-Francisco Syncope Rule. ${ }^{3}$ Patients investigated for conditions other than syncope 